\title{
Microindentation technique as a tool for investigating the development of order in PET under uniaxial stress
}

\author{
Ahmed I. Abou-Kandil • A. Flores - F. J. Balta Calleja • \\ A. H. Windle
}

Received: 21 September 2007 / Accepted: 11 February 2008 / Published online: 28 February 2008

(C) Springer Science + Business Media B.V. 2008

\begin{abstract}
The development of microhardness during annealing was used as a tool to follow the development of the liquid crystalline transient mesophase during the crystallization of uniaxially oriented polyethylene terephthalate (PET). 2D X-ray diffraction was used to characterize the different stages of the crystallization process. We were able to separate those stages by quenching into air. Microindentation hardness experiments were done in real time where samples were heated with their ends fixed on a specially developed stage and microhardness was measured simultaneously. The oriented samples examined exhibit a clear difference in behavior from isotropic samples that mainly lack the existence of such an ordered mesophase. The mesophase clearly has a reinforcement effect on the whole polymer matrix that leads to an increase in hardness value with annealing of the oriented PET films. Microindentation hardness is shown to be a versatile tool to detect the existence of the liquid crystalline transient mesophase. It is also efficient in
\end{abstract}

A. I. Abou-Kandil • A. H. Windle

Department Of Materials Science and Metallurgy,

University of Cambridge,

Pembroke Street,

Cambridge CB2 3QZ, UK

A. Flores $\cdot$ F. J. Balta Calleja

Instituto de Estructura de la Materia, CSIC,

Serrano, 119,

28006 Madrid, Spain

Present address:

A. I. Abou-Kandil $(\square)$

Max-Planck Institute for Polymer Research,

Ackermannweg 10,

Mainz 55128, Germany

e-mail: aia_23@yahoo.co.uk comparing and explaining results obtained by wide angle $\mathrm{X}$-ray scattering.

Keywords Microhardness · PET - Crystallization .

Mesophase · Order · Uniaxial stress

\section{Introduction}

The current understanding of polymer crystallisation is moving towards a picture in which ordering takes place in stages involving one or more intermediate stages, broadly termed as mesophases. Although the concept is quite old, having been recognised already by Ostwald in his rule of stages in the late 19th Century, it has only gathered recognition in polymer crystallisation field in recent years.

Early models of the staged ordering in polyethylene terephthalate (PET) described by Bonart [1] remained largely unnoticed until recently. In 1995 Imai et al. [2] suggested an initial ordering of unoriented samples of PET into nematic like regions in a spinodal decomposition process followed by crystallisation.

Auriemma et al. [3] and Nicholson et al. [4], attempted to model the structure of the PET mesophase. They suggested the existence of two stable structures for PET. In one structure the PET chains are fully extended, corresponding to the usual triclinic form and an alternative conformation, where the dihedrals between the $\mathrm{CO}-\mathrm{O}-\mathrm{C}-\mathrm{C}$ are rotated by about $80^{\circ}$.

Studies published by Welsh et al. [5, 6], indicated the ability of presence of the mesophase in the 50/50 PET/ polyethylene naphthalate (PEN) copolymer fibres drawn at $100{ }^{\circ} \mathrm{C}$ to draw ratios (DR) of 6 and 10 . Also recent studies by Mahendrasigam et al. [7] and Blundell et al. [8] conducted on PET, PEN and 50\% PET/PEN random 
copolymer have also confirmed the existence of such a mesophase.

Abou-Kandil et al. [9] studied in detail the development of microstructure during the annealing of oriented PET films using X-ray scattering techniques. These results concluded that there is a uniform global change taking place over the entire sample during the transition between oriented amorphous and oriented crystalline states. The Wide Angle X-ray Scattering (WAXS) results discussed indicated the presence of an intermediate liquid crystalline phase prior to crystallization. The mesophase was classified as having a smectic-A type order. On the other hand the small Angle X-ray Scattering (SAXS) results from the mesophase suggested the presence of fibrillar arrangement of the polymer chains in the direction of the draw. This was represented by the initial fibre streaking, but it gave no evidence of density variation which would indicate the presence of significant microstructure. However, once crystallization started on annealing above the glass transition temperature, the SAXS showed very clear evidence of microstructure which then developed. We also studied the morphology using scanning electron microscope [10] and used molecular modelling to validate the structure of the mesophase and compare the resulting diffraction patterns it to the experimental data [11].

Asano et al. [12] have used hardness measurements to distinguish between the different phases occurring during the crystallization of oriented PET. In their experiments all measurements have been done at room temperature and it showed that the hardness of PET increased by increasing the annealing temperature and that the indentation anisotropy decreases when PET samples are annealed at temperatures above glass transition $\left(T_{g}\right)$. The aim of our present paper is to examine the microhardness behaviour of the oriented glassy PET, in real time, as a function of temperature. We intend to contribute to the understanding of the role of the transient mesophase in the crystallization process.

\section{Microhardness of oriented polymers}

Microindentation using a point indenter involving a deformation on a very small scale is one of the simplest methods for determining the microhardness, $H$, of a material. The method uses a diamond pyramid indenter that penetrates the surface of the specimen upon application of a given load at a constant rate. A convenient measure of $H$ may be obtained by dividing the peak contact load, $P$, by the projected area of deformation, $A, H=P / A$. Detailed description of the measurement will be explained in "Experimental" below.

Uniaxial mechanical deformation provokes drastic changes in the indentation pattern of drawn polymers. The newly created fibre structure consists of highly aligned microfibrils within the microfibrillar stacks of crystal blocks perpendicularly oriented to the draw direction. The aligned microfibrils act as cross-links for the molecules, bridging adjacent crystalline layers.

Two well-defined hardness values can be defined due to orientation $[12,13]$. One value, maximum $\left(H_{/ /}\right)$, derived from the indentation diagonal parallel to the fibre axis $\left(d_{/ /}\right)$, the second one, minimum $\left(H_{\perp}\right)$, is deduced from the diagonal perpendicular to it $\left(d_{\perp}\right)$. The former value is in fact not a physical measure of hardness, but corresponds to an instant elastic recovery of the fibrous network in the draw direction. The latter value defines the plastic component of the oriented material. From the morphology of the fibrous structure of the deformed polymer one concludes that the dominant deformation modes of the drawn polymer under the stress field of the indenter involve:

1. Sliding motion of fibrils and microfibrils, which are sheared and displaced normal to the fibre axis under compressive load.

2. Buckling of fibrils parallel to the fibre axis. Since the shape of the indentation must conform with that of the diamond indenter while the load is applied, the anisotropy observed must arise instantly upon load removal because of greater elastic recovery of the fibrillar network with strained molecules along the fibre axis where the stress is largest.

Indentation anisotropy, $\Delta H$, is defined as:

$\Delta H=1-\frac{H_{\perp}}{H_{/ /}}$

\section{Experimental}

Microhardness measurements

PET samples having three different molecular weights are used in the micoindentation experiments. The intrinsic viscosity of PET used is $0.9,0.65$ and 0.45 as given by the supplier, ICI. The molecular weight of the polymer was found to be about $82,000,50,000$ and $28,000 \mathrm{~g} / \mathrm{mol}$ respectively. The molecular weight was calculated using Kuhn-Mark-Houwink equation [14]:

$[\eta]=\kappa M_{v}^{\alpha}$

The constants for PET are $\alpha=0.695$ and $\kappa=5.2 \times$ $10^{-4} \mathrm{ml} / \mathrm{g}$ [15].

Different oriented amorphous polymer samples were mounted on a hot stage (Mettler FP52) under a microindenter with a square diamond tester. The samples were fixed on an aluminium stage on top of the heating stage by 
means of a double-sided temperature resistant adhesive tape to ensure the surface of the sample remains flat during the experiment. The ends of the samples were also fixed with double screws at the edge of the aluminium stage to prevent the oriented sample from shrinkage.

Microindentation experiments were carried out using a Vickers square based diamond pyramid approximately $200 \mu \mathrm{m}$ in height. The included angles between opposite faces, $2 \alpha$, equals $136^{\circ}$. A typical load of $0.05 \mathrm{~N}$ using this indenter would produce a penetration depth, $h_{p}$, of about 3$4 \mu \mathrm{m}$ in PET samples. Vickers hardness is calculated according to the expression:

$H=k \frac{P}{d^{2}}$

Where $P$ is the load applied, $d$ is the measured diagonal of the residual impression and $k$ is a geometric constant. A value of $k=1.854$ is used when $P$ is in Newton and $d$ is in millimeter to give $H$ in molar pascal.

A loading cycle of $0.1 \mathrm{~min}$ is used to minimise creep [16]. The sample thickness was always seven to ten times greater than the measured diagonal values in order to make sure that the measurements taken are the real hardness of the samples without interference of the surface on which the samples were fixed [17]. At least three different loads for each sample were used that verifies the above condition, ranging from $0.147^{\circ} \mathrm{N}$ to $2.942^{\circ} \mathrm{N}$. This was necessary during the microindentation measurement to make sure the value of $\frac{P}{d^{2}}$ was maintained constant and to correct for the instantaneous elastic recovery of the polymer at high temperatures if necessary [18].

The temperature at the surface of the sample was calibrated against several standards. Sputtering of the sample surface using gold was performed to improve optical contrast during the indentation.

\section{X-ray diffraction}

Five different samples have been prepared from $500 \mu \mathrm{m}$ thick PET films. Each sample was chosen to represent a specific stage observed in the WAXS analysis described in references [9-11]. These stages are: amorphous, oriented amorphous, mesophase, oriented crystalline and oriented crystalline annealed.

In order to prepare each microstructural stage, each sample was drawn to a specific draw ratio at a given temperature and then quickly quenched to air or annealed with the ends fixed at a given temperature. Figure 1 illustrates how each sample was prepared and shows a diffraction 2D diffraction pattern for each sample after preparation.

The X-ray measurements were done on a Philips PW $1729 \mathrm{X}$-ray generator using a graphite filtered copper $K_{\alpha} \mathrm{X}$ ray tube producing radiation with a wavelength of $1.54 \AA$.
The measurements were made in transmission mode, using a CCD camera as a detector. The sample-to-film distance was calibrated using silicon powder that gives a sharp (100) ring at a $2 \theta$ value of $28.442^{\circ}(d$-value of $3.135 \AA$ ).

\section{Results and discussion}

Microindentation hardness has been shown to be a valuable tool capable of distinguishing between the amorphous, smectic and crystalline structure of annealed cold drawn PET films after cooling the sample at room temperature [12] as well as unoriented PET and PEN samples [19, 20]. What we are interested in now is to perform the experiments in real time as a function of temperature and time so that we understand the effect of the intermediate liquid crystalline mesophase on the crystallization process. The results to be obtained here are to be interpreted in accordance with the X-ray scattering results obtained [9].

Figure 1 shows the key stages occurring on uniaxially drawing PET at either $40{ }^{\circ} \mathrm{C}$ or room temperature to a draw ratio of 3.5 and subsequently annealing the sample with its ends fixed. It is clear from the figure that the starting sample is amorphous. This is indicated by the obvious diffuse amorphous halo indicating the absence of crystallinity in the sample, as shown in the 2D WAXS patterns in Fig. 1a. Drawing leads to the orientation of the polymer in the direction of the draw, and the amorphous halo is concentrated on the equator as shown in Fig. $1 \mathrm{~b}$.

Subsequent annealing leads to the formation of the smectic mesophase, Fig. 1c. The mesophase acts as a precursor to triclinic crystals [5, 6, 9-11]. The key aspects of Fig. 1c are the high degree of orientation and the almost complete absence of crystallisation. The presence of a sharp meridional peak at a position equivalent to $10.42 \AA$ and the absence of the crystalline order in the interchain packing means that the sample has order which could well be classified as smectic A. A more detailed explanation of the development of structure using X-ray diffraction can be found in references [9-11].

Figure $1 \mathrm{~d}$ represents the oriented crystalline sample drawn to a draw ratio of 3 where it is obvious that there is a hint of splitting starting to take place in the equatorial reflections indicating the existence of horizontal register between the chains and low, two-dimensional, crystallinity.

Figure 1e on the other hand obtained by annealing the oriented crystalline sample at $190{ }^{\circ} \mathrm{C}$ for $24 \mathrm{~h}$ shows distinct splitting of the equatorial reflection into three distinct reflections indicating lateral packing of the chains. The clear first layer lines indicate the development of threedimensional crystalline structure.

It is of extreme importance also to compare those results with the ones obtained for isotropic polymer samples [18, 21]. 
Fig. 1 Wide angle X-ray scattering patterns showing the key staged of development of microstructure during drawing of amorphous PET at $40{ }^{\circ} \mathrm{C}$ to a draw ratio of 3.5 and then subsequent annealing of the sample with its ends fixed to prevent shrinkage. a Amorphous, b Oriented Amorphous, c Mesophase, d Oriented Crystalline, e Oriented Annealed
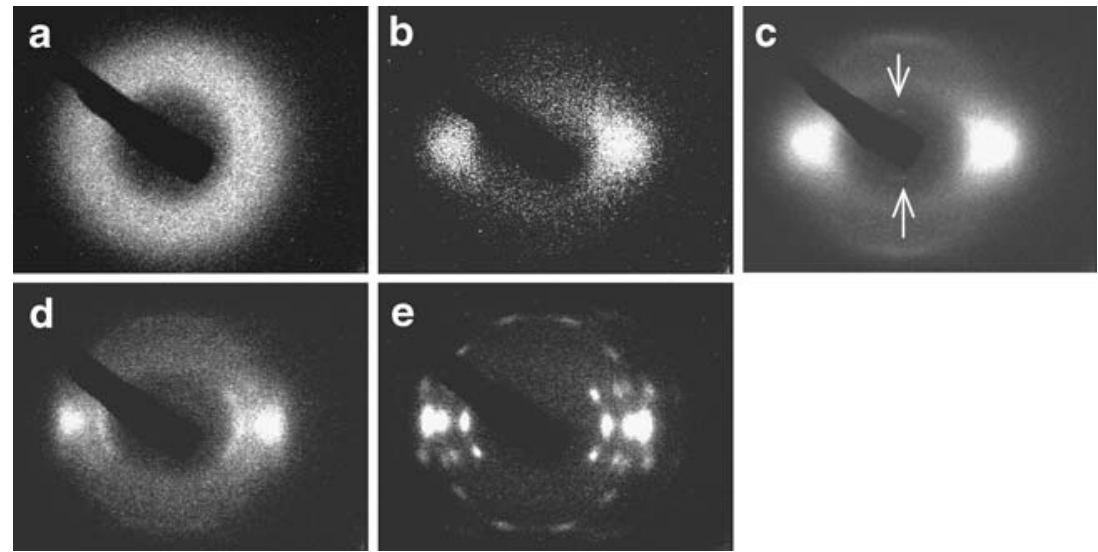

Development of microhardness with temperature

Oriented amorphous samples were clamped on the Aluminium heating stage as explained above; the temperature was raised at $2{ }^{\circ} \mathrm{C} \mathrm{min}^{-1}$ and left to equilibrate every $5{ }^{\circ} \mathrm{C}$ for $3 \mathrm{~min}$, afterwards the measurements of both $d_{/ /}$and $d_{\perp}$ were made.

\section{Development of microhardness in oriented PET}

Measuring the microindentation hardness for oriented amorphous PET samples drawn at $40{ }^{\circ} \mathrm{C}$ during annealing revealed the behaviour shown in Fig. 2. It is clear that $\mathrm{H}_{/ /}$ values are greater than those of $\mathrm{H}_{\perp}$. This is the consequence of the instant elastic recovery of the fibrils after removal of the indenter, and therefore contributes to the smaller indentation diagonal in the direction perpendicular to the fibre axis. The microhardness values in this case appear to be unchanged until $65{ }^{\circ} \mathrm{C}$, then they start to increase abruptly until the temperature reaches $100{ }^{\circ} \mathrm{C}$, afterwards the increase is less steep and appears to level off. The behaviour is the same for both $\mathrm{H}_{/ /}$and $\mathrm{H}_{\perp}$. As expected, the microhardness values increase by increasing the molecular weight of the PET samples, which was also observed in an earlier study [14].

Similar behaviour is observed for samples that were drawn at room temperature, shown in Fig. 3. The only difference is that microhardness values in this case do not level off at around $120^{\circ} \mathrm{C}$ as observed for samples drawn at $40{ }^{\circ} \mathrm{C}$. This might be because drawing the samples at lower temperature decreases the ability of the polymer chains to align in the direction of the draw. Consequently, they need longer times and higher temperatures to reach an equilibrium state.

Figure 4 illustrates the variation of indentation anisotropy as a function of temperature for the series of PET samples; anisotropy is a measure of the elastic recovery of the material as discussed above. The first finding in Fig. 4 is the unusually high $\Delta H$ values for the samples investigated compared to those reported for PET in earlier studies [12]. However, in those experiments all the measurements were made at room temperature after annealing the samples at certain temperatures. As an average behaviour for all three PET samples under consideration, the values of $\Delta H$ start at about 0.4 and then increase gradually with temperature until they reach values as high as 0.6 at $80{ }^{\circ} \mathrm{C}$ $\left(\approx T_{g}\right.$ of PET) before starting to drop to values comparable to the initial ones.

\section{Influence of temperature on microhardness}

From Figs. 3 and 4 four regions of $\mathrm{H}_{/ /}$and $\mathrm{H}_{\perp}$ values can be observed which correlate to various structures as the temperature is increased:

1. Temperatures $\leq 65{ }^{\circ} \mathrm{C}$ : from the WAXS results presented in an earlier study [9] it is clear that the smectic mesophase is present at these temperatures after orientation of the sample. The values of both $\mathrm{H}_{/ /}$and $\mathrm{H}_{\perp}$ appear to be unaffected by the structural changes occurring at these temperatures leading to the approximate levelling of the microhardness values. This behaviour is comparable with that observed for isotropic PET below $T_{g}$, where the hardness values remain approximately unchanged with increasing temperature $[19,21]$.

2. $65{ }^{\circ} \mathrm{C}<$ Temperature $\leq 80{ }^{\circ} \mathrm{C}$ : the hardness values increase steadily. Within this temperature range the amount of mesophase present is sufficient to produce an appreciable increase in hardness by reinforcing the structure.

3. $80{ }^{\circ} \mathrm{C}<$ Temperature $\leq 100{ }^{\circ} \mathrm{C}$ : the hardness values increase steeply and this is associated with the 

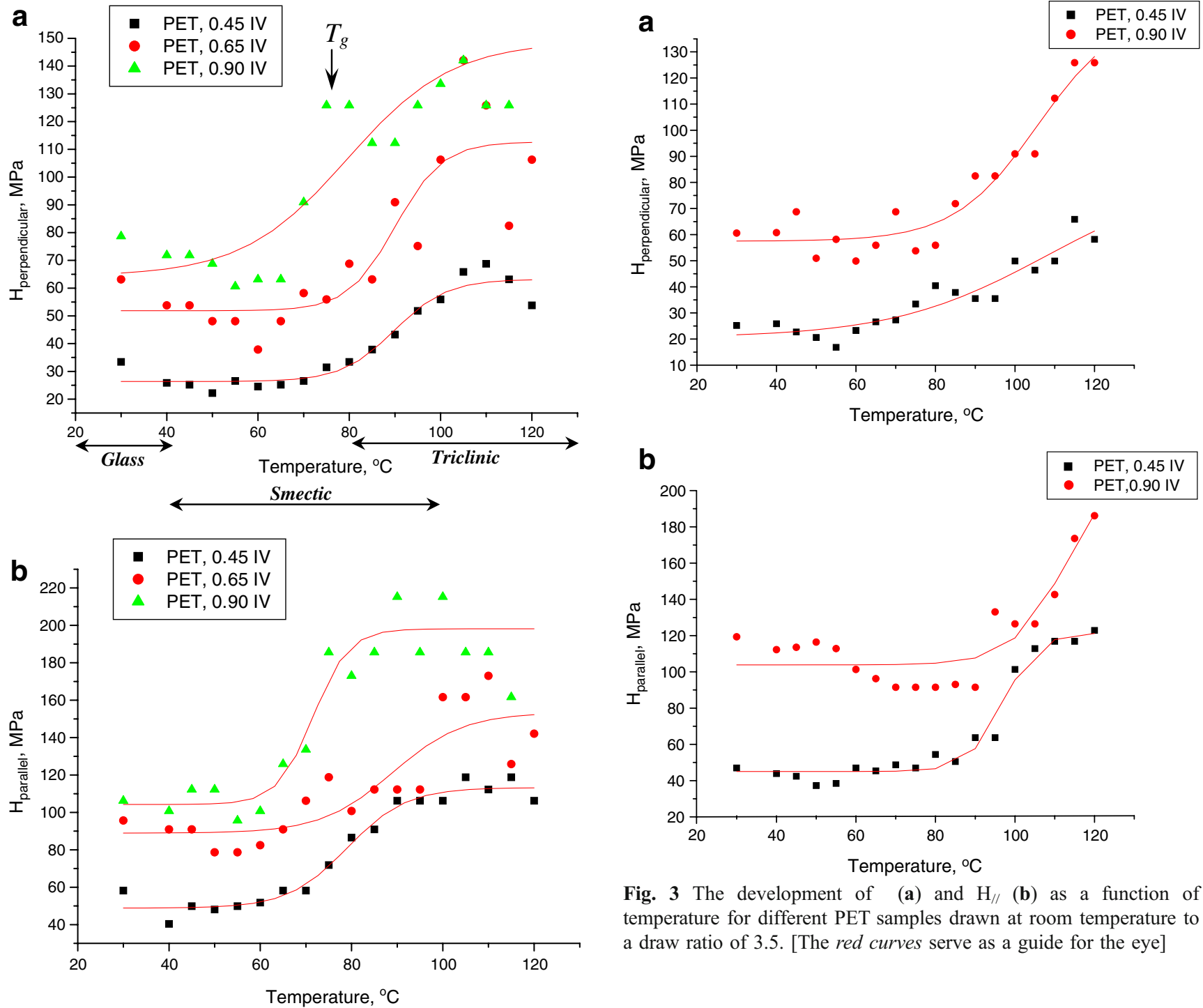

Fig. 3 The development of (a) and $\mathrm{H}_{/ /}$(b) as a function of temperature for different PET samples drawn at room temperature to a draw ratio of 3.5. [The red curves serve as a guide for the eye]

Fig. 2 The development of (a) and $\mathrm{H}_{/ /}$(b) as a function of temperature for different PET samples drawn at $40{ }^{\circ} \mathrm{C}$ to a draw ratio of 3.5. [The red curves serve as a guide for the eye]

formation of the triclinic crystals and the start of the disappearance of the smectic domains.

4. Temperature $\geq 100{ }^{\circ} \mathrm{C}$ : the smectic phase completely disappears and a three dimensional crystalline order develops as discussed in a previous publication [9]. This is associated with the approximate levelling of hardness values for samples drawn at $40{ }^{\circ} \mathrm{C}$ or continuing to increase in high molecular weight PET drawn at room temperature. These results are in contrast to those reported for isotropic PET [19, 21], where the hardness values drop abruptly after $T_{g}$ due to the softening of the material.

From the above results it can be clearly seen that at low temperatures $\left(\leq T_{g}\right)$ the increase in hardness could be

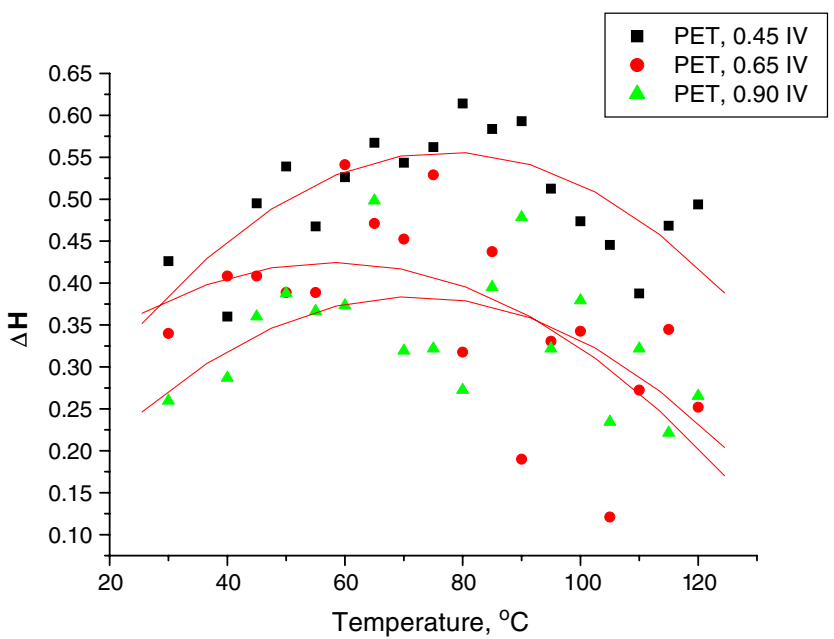

Fig. 4 The development of $\Delta H$ as a function of temperature for different PET samples drawn at $40{ }^{\circ} \mathrm{C}$ to a draw ratio of 3.5. [The red curves serve as a guide for the eye] 
associated with the increase in the smectic order and the possible densification of the smectic domains. On the other hand, at temperatures above $T_{g}$ the increase in hardness appears to be associated with the increase in crystallinity, and it levels off with the levelling of the crystallinity value.

\section{Influence of temperature on indentation anisotropy}

The results shown in Fig. 4 show that in all three PET samples the indentation anisotropy passes through a maximum at about $T_{g}$ of PET i.e. at around the temperature where the amount of mesophase present in the sample is a maximum.

The initial increase in the value of $\Delta H$ with temperature might be due to the increase in the value of $\mathrm{H}_{\perp}$, due to the increase in the mesophase content, it reaches a maximum when the amount of mesophase is maximum and then starts to decline again due to the decrease in the elastic recovery of the material in the chain direction i.e. the decrease of the value of $\mathrm{H}_{/ /}$. Similar behaviour was observed above $T_{g}$ for PET in an earlier study [12].

\section{Kinetics of mesophase and crystallisation development}

The polymer samples were held at constant temperature with their ends fixed to prevent sample shrinkage at high temperature. The sample temperature was raised at $2{ }^{\circ} \mathrm{C} \mathrm{min}^{-1}$ until the temperature at the surface of the sample reached the desired. This slow ramping of the temperature prevents the temperature from over shooting. The hardness was then monitored until a plateau was reached in the hardness value, or a constant behaviour was observed for more than 30 min.

In the next few sections only reference to $\mathrm{H}_{\perp}$ will be made as it represents the plastic deformation mode within the fibrous structure of the material and is related to the inter-chain stacking necessary for the formation of the smectic mesophase and/or the triclinic crystals, whereas $\mathrm{H}_{/ /}$ represents the deformation taking place in the direction of the draw.

Development of mesophase and crystallinity in PET

Figure 5a shows $\mathrm{H}_{\perp}$, in MPa, versus time, in minutes, for different PET samples drawn to a draw ratio of 3.5. It is clear from the figure that all samples show the same behaviour with a steep increase in hardness followed by a plateau. The time needed to reach the plateau increases with increasing the molecular weight of PET. This time is $20 \mathrm{~min}$ for PET $0.45 \mathrm{IV}, 28,000 \mathrm{~g} \mathrm{~mol}^{-1}, 30 \mathrm{~min}$ for PET $0.65 \mathrm{IV}, 50,000 \mathrm{~g} \mathrm{~mol}^{-1}$ and $40 \mathrm{~min}$ for PET $0.90 \mathrm{IV}$, $82,000 \mathrm{~g} \mathrm{~mol}^{-1}$. It can be concluded that after this period
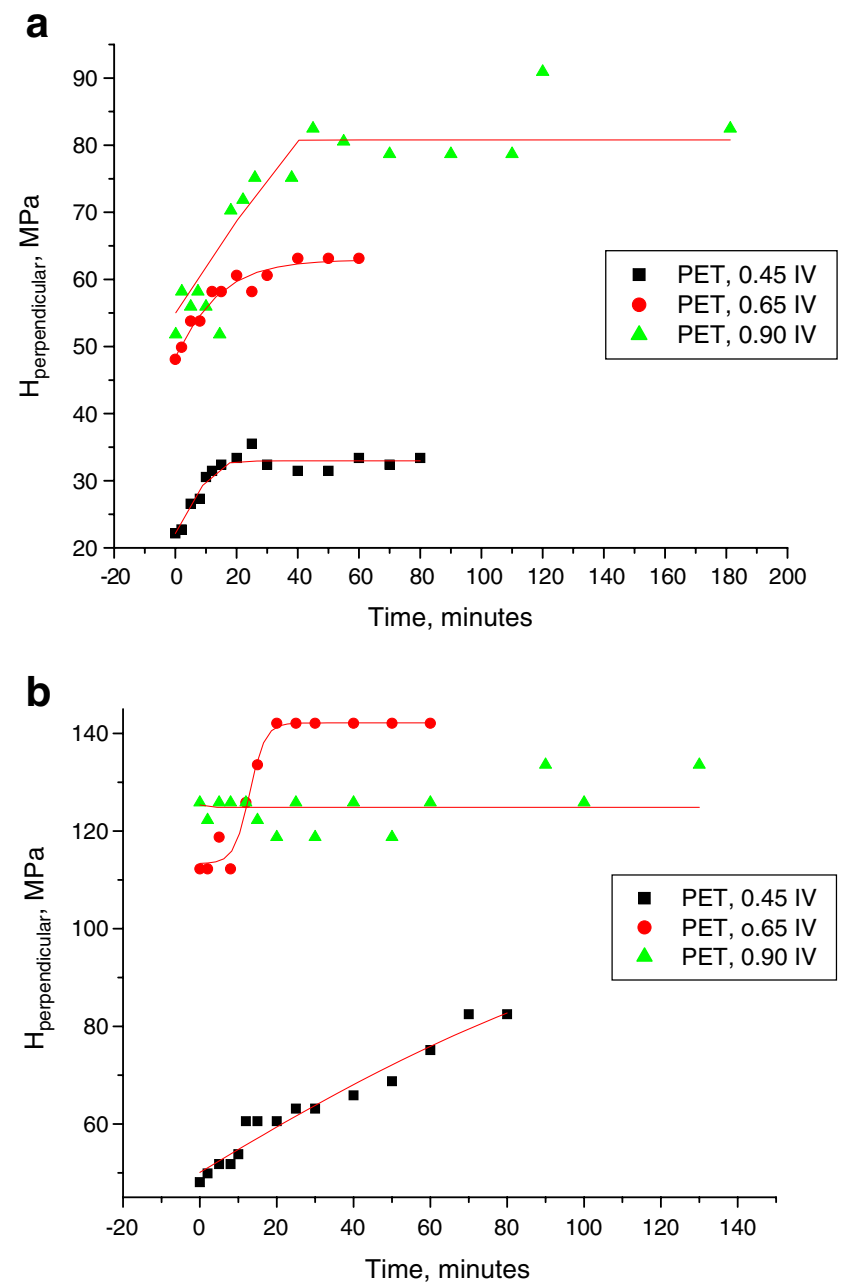

Fig. 5 The development of as a function of time for different PET samples at $65{ }^{\circ} \mathrm{C}(\mathbf{a})$, smectic phase region, and $115^{\circ} \mathrm{C}(\mathbf{b})$, triclinic region. [The red curves serve as a guide for the eye]

the amount of mesophase in the sample reaches maximum and this leads to the levelling off of the hardness values measured. It is relevant to note here that $65{ }^{\circ} \mathrm{C}$ is not high enough to induce crystallisation and this is confirmed by the X-ray measurements reported earlier [9].

Figure $5 \mathrm{~b}$ shows the behaviour of the different PET samples at $115{ }^{\circ} \mathrm{C}$, which is above $T_{g}$ of PET so crystallisation can take place, and accordingly the kinetics of the crystallisation can be monitored. Interestingly, the behaviour of the three samples of PET is different. The lowest molecular weight PET sample shows a steady increase in hardness with time. The longer time needed by 0.45 IV PET to reach the plateau may be because it needs more time for densification to take place. On the other hand, the highest molecular weight PET seems to have reached the plateau of crystallinity already; the high molecular weight might have led to crystallisation to be completed at a lower temperature. The intermediate molecular weight PET requires $20 \mathrm{~min}$ to reach the 
plateau, which means that the crystallinity increases gradually with a maximum value is attained after about $20 \mathrm{~min}$ at $115^{\circ} \mathrm{C}$.

\section{Conclusions}

Although PET is regarded as one of the most well known polymers; it is still regarded as a hot point of research inducing recent publications $[22,23]$. From the results obtained in this paper it can be concluded that:

1. The microhardness of cold drawn PET samples exhibits an initial plateau at the beginning of annealing and then increase steeply as the material becomes reinforced, first by the presence of the smectic mesophase, and then the triclinic crystals. The mesophase in this case acts as a strong molecular network that reinforces the material and leads to increase in hardness values. This is opposed to the fact that in case of unoriented PET the hardness values decrease sharply at temperatures above $T_{g}$. Afterwards the hardness levels off when the maximum crystallinity is attained.

2. Indentation anisotropy $(\Delta H)$ is directly proportional to the amount of mesophase and oriented material present in the sample. It seems to be a valuable and effective measure of the degree of orientation of the sample. PET clearly exhibits maximum $\Delta H$ values at maximum degree of orientation.

3. The microhardness technique appears to be a valuable tool for studying the kinetics of the development of the mesophases, and the crystallinity of oriented polymer samples, this comes in agreement with recent publications relating both techniques [24].

\section{References}

1. Bonart R (1966) Koll Z Z Polym 210:16 213:1 (1966), and 231:438 (1967)

2. Imai M, Kaji K, Kanaya T, Sakai Y (1995) Phys Rev B 52 (17): 12696

3. Auriemma F, Corradini P, DeRosa C, Guerra G, Petraccone V, Bianchi R, DiDino G (1992) Macromolecules 25:2490

4. Nicholson TM, Davis GR, Ward IM (1994) Polymer 35(20):4259

5. Welsh GE, Blundell DJ, Windle AH (1998) Macromolecules 31:7562

6. Welsh GE, Blundell DJ, Windle AH (2000) J Mater Sci 35:5225

7. Mahndasigam A, Martin C, Fuller W, Blundell DJ, Oldman RJ, Harvie JL, MacKerron DH, Riekel C, Engstrom P (1999) Polymer 40:5553

8. Blundell DJ, Mahendrasingam A, Martin C, Fuller W (2000) J Mater Sci 35:5057

9. Abou-Kandil AI, Windle AH (2007) Polymer 48(17):5069

10. Abou-Kandil AI, Windle AH (2007) Polymer 48(16):4824

11. Abou-Kandil AI, Windle AH (2007) Macromolecules 40(18):6448

12. Asano T, Balta Calleja FJ, Flores A, Tanigaki M, Mina MF, Sawatari C, Itagaki H, Takahashi H, Hatta I (1999) Polymer 40:6475

13. Balta-Calleja FJ (1985) Adv Polym Sci 66:117

14. Vanderdonckt C, Krumova M, Balta Calleja FJ, Zachmann HG, Fakirov S (1998) Colloid Polym Sci 276:138

15. Berkowitz S (1984) J Appl Polym Sci 29:4353

16. Verma RK, Hsiao BS (1996) Trends Polym Sci 4:312

17. Balta-Calleja FJ, Fakirov S (2000) Microhardness of polymers. Cambridge University Press, Cambridge

18. Balta Calleja FJ, Rueeda DR, Porter RS, Mead WT (1980) J Mater Sci 15:765

19. Santa Cruz C, Balta Calleja FJ, Zachmann HG, Stribeck N, Asano T (1991) J Polym Sci Part B Polym Phys 29:819

20. Kajaks J, Flores A, Garcia Gutierrez MC, Rueda DR, Balta Calleja FJ (2000) Polymer 41:7769

21. Balta Callega FJ, Santa Cruz C, Asano T (1993) J Polym Sci Part B Polym Phys 31:557

22. Hsu G-H, Yang Y-N, Yu TL, Lin H-L (2006) J Polym Res 13:361

23. Ren M, Zhang Z, Wu S, Wei J, Xiao C (2006) J Polym Res 13:9

24. Uchiyama T, Suyama M, Alam MM, Asano T, Henning S, Flores A, Balta-Calleja FJ, Mina MF (2007) Polymer 48:542 